\title{
Noise measurement in amplifying system for radiation detectors
}

\begin{abstract}
Electronic noise is a critical parameter especially for electronic devices which deal with low level input signals such as preamplifier-shaper chains in nuclear instrumentation. It degrades the capability of the amplifying system as a radiation measurement device in terms of detection level, energy resolution and event measurement. In this paper, we propose an experimental approach to noise measurement in amplifying systems for radiation detectors. The experimental results are verified with theoretical computation and validated with an established experimental method. The results are in good agreement with theoretical and established methods.
\end{abstract}

Keyword: Noise; Amplifying system; Radiation detectors 